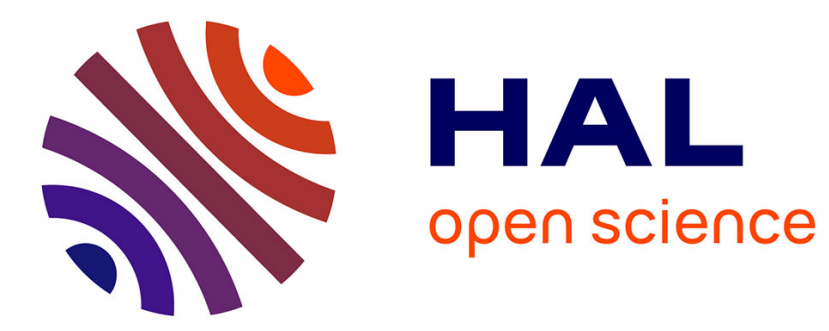

\title{
Les lasers moléculaires ultraviolets à excitation électronique transverse
}

\author{
J.-P. Girardeau-Montaut
}

\section{To cite this version:}

J.-P. Girardeau-Montaut. Les lasers moléculaires ultraviolets à excitation électronique transverse. Revue de Physique Appliquée, 1979, 14 (2), pp.311-322. 10.1051/rphysap:01979001402031100 . jpa00244600

\section{HAL Id: jpa-00244600 https://hal.science/jpa-00244600}

Submitted on 1 Jan 1979

HAL is a multi-disciplinary open access archive for the deposit and dissemination of scientific research documents, whether they are published or not. The documents may come from teaching and research institutions in France or abroad, or from public or private research centers.
L'archive ouverte pluridisciplinaire HAL, est destinée au dépôt et à la diffusion de documents scientifiques de niveau recherche, publiés ou non, émanant des établissements d'enseignement et de recherche français ou étrangers, des laboratoires publics ou privés. 


\title{
Les lasers moléculaires ultraviolets à excitation électronique transverse
}

\author{
J.-P. Girardeau-Montaut \\ Laboratoire Universitaire de Bourges, Université d'Orléans, Groupe de Physique des Lasers, \\ 63, avenue du Maréchal-de-Lattre-de-Tassigny, 18000 Bourges, France
}

(Reçu le 30 juin 1978, révisé le 5 décembre 1978, accepté le 11 décembre 1978)

\begin{abstract}
Résumé. - Les plus récents développements des lasers moléculaires ultraviolets, à excitation électronique transverse sont présentés et analysés. Ces lasers peuvent être regroupés en deux familles caractérisées par le type de transition à partir de laquelle a lieu l'émission stimulée : transitions entre deux états liés et transitions entre un état supérieur lié et un état inférieur répulsif. Le second type offre des possibilités et laisse prévoir des performances supérieures à celles du premier, mais celles-ci ne sont pas encore toutes exploitées. Est également présenté l'état actuel de la technologie de ces lasers. Son développement permet de disposer de montages bien adaptés à un certain nombre d'applications. Un autre avantage est constitué par la possibilité de disposer de plusieurs raies lasers par simple changement du milieu gazeux excité. Enfin, les rendements d'émission élevés obtenus dans plusieurs cas expliquent l'intérêt porté sur les deux plans : théorique et expérimental.
\end{abstract}

\begin{abstract}
The more recent developments of ultraviolet molecular laser, with transverse electronic pumping, are presented here. It is show that two models of electronic transitions : bound-bound and bound-unbound transitions, are in the beginning of the most part of intense ultraviolet laser lines. The second model offer some possibilities of stimulated emission which are not all worked. We point out, in the last part of this paper, some molecules from which laser lines should be possible. So, we analyze the state of the laser technology development. This has lead to realize several systems from which it is possible to have numerous laser lines with just changing the gases. As a practical consequence, may applications exist.
\end{abstract}

1. Introduction. - Depuis la mise en évidence expérimentale, par Maiman [1] en 1960, du phénomène d'émission stimulée dans le domaine optique, les développements des lasers n'ont cessé de prendre de l'importance, à la fois en ce qui concerne la connaissance fondamentale des phénomènes physiques et de la matière, des technologies associées et des applications. La plus grande part de ces développements intéresse cependant les régions spectrales de l'infrarouge (IR) et du visible (V), ceci malgré l'intérêt que présente le domaine de l'ultraviolet (UV). Citons par exemple : l'étude détaillée de la structure électronique des atomes et des molécules, la diffusion Raman, la séparation isotopique et les autres synthèses chimiques, l'application aux communications terrestres (en particulier la région autour de $2700 \AA$ à cause de la couche d'ozone située à haute altitude [2]) et spatiales (principalement en dessous de $2000 \AA$ [3]), enfin l'application à la fusion thermonucléaire [4], les lasers UV pouvant permettre le chauffage de plasmas de haute densité si l'on s'en tient au fait que le taux d'absorption du Bremsstrahlung inverse est de l'ordre de $\lambda^{-2}$.

Il y a plusieurs raisons à cette situation particulière. Tout d'abord, comme l'avaient noté, dès l'origine,
Schawlow et Townes, il est d'autant plus difficile d'obtenir un effet laser que la longueur d'onde $\lambda \mathrm{du}$ rayonnement est courte. En effet, le gain optique $g$, au centre de la raie d'émission, a pour expression :

$$
g=\left(\frac{4 \pi^{2}}{3 \hbar c}\right) \mu^{2}\left(\frac{n_{2}}{g_{2}}-\frac{n_{1}}{g_{1}}\right) \frac{v}{\Delta v}
$$

dans laquelle : $\mu$ représente l'élément de matrice de transition, $n_{1}$ et $n_{2}$ sont les densités de population des niveaux inférieur et supérieur laser, $g_{1}$ et $g_{2}$ étant les dégénérescences respectives de ces niveaux; $v$ désigne la fréquence et $\Delta v$ la largeur de raie de la transition. En première approximation, $\left(\frac{n_{2}}{g_{2}}-\frac{n_{1}}{g_{1}}\right)$ est proportionnel à $v^{-3}$, c'est-à-dire à $\lambda^{3}$. D'autre part, dans le domaine de fréquences auquel nous portons attention, il a été démontré que la cause principale d'élargissement de raie dans les gaz est du type Doppler, c'est-àdire que $\Delta v$ est proportionnelle à $v$. Les autres causes d'élargissement : par collisions [5], radiatif, par effet Stark [6] ou par effet Auger [7] ont en général des effets beaucoup moins importants, surtout dans le cas 
d'une transition résonnante entre deux états liés. Dans ces conditions $g$ est proportionnel à $v^{-3}$.

Malgré cela, l'expérience a montré qu'il était possible d'obtenir des émissions stimulées dans l'UV à partir d'atomes ou de molécules [8]. Un très grand nombre de transitions lasers ont en effet été observées entre $155 \mathrm{~nm}$ et $400 \mathrm{~nm}$ à partir d'atomes ionisés principalement. Les densités d'énergie émise et les rendements d'émission observés sont cependant beaucoup plus faibles que ceux obtenus à partir des molécules diatomiques $\left(\mathrm{N}_{2}, \mathrm{H}_{2}, \mathrm{D}_{2}, \mathrm{HD}, \mathrm{CO}, \mathrm{XeF}, \mathrm{ArF}\right.$, $\mathrm{KrF}, \mathrm{ArCl}, \mathrm{KrCl}, \mathrm{XeCl}, \mathrm{Ar}_{2}, \mathrm{Xe}_{2}, \mathrm{Kr}_{2}, \mathrm{~F}_{2}, \mathrm{Cl}_{2}, \mathrm{Br}_{2}$ et $\mathrm{I}_{2}$ ).

Dans le cas des molécules, étant donné que l'énergie $\mathrm{h} v$ des rayonnements UV est supérieure à $3 \mathrm{eV}$, il ne peut s'agir que de transitions entre états électroniques. Toutes les transitions entre niveaux vibrationnels ou rotationnels d'un même état électronique sont donc exclues. Compte tenu d'autre part des règles de sélection, le nombre de molécules susceptibles d'être le siège d'une émission UV stimulée est limité.

D'autres faits viennent encore réduire ces possibilités. On observe actuellement que les émissions UV lasers les plus énergétiques sont obtenues à partir de matériaux en phase gazeuse. Or, les molécules gazeuses présentent généralement des sections efficaces d'émission stimulée plus grandes que celles rencontrées généralement dans les solides et les liquides, étant donné les faibles largeurs de raie $\Delta v$. Cette caractéristique accroît le risque d'émission super-radiante et d'oscillations parasites et réduit le taux d'inversion de population admissible. D'autre part, le phénomène de désexcitation collisionnel devient important lorsque la pression du gaz est élevée. Cette autre donnée est la cause de pertes d'énergie importantes qui limitent le rendement total d'émission. Il est à noter cependant que la phase gazeuse offre conjointement plusieurs avantages : le seuil de destruction d'un gaz soumis à de grandes densités d'énergie radiative est supérieur à celui des solides ou des liquides et son remplacement est souvent plus aisé ; les pertes d'énergie par diffusion sont plus faibles; la diffraction du faisceau lumineux peut être limitée plus aisément et enfin, il est possible d'obtenir des taux de répétition beaucoup plus élevés [9] dans le cas d'émissions pulsées.

Une autre cause de limitation de ce développement est due au petit nombre des modes d'excitation. L'absence, à l'origine, de sources de rayonnement UV quasi monochromatiques et de grande intensité ainsi que l'emploi efficace de l'excitation électronique pour la création de nombreuses sources UV traditionnelles (lampes à hydrogène, à deutérium, à vapeur de mercure, etc.) ont conduit tout naturellement à développer les technologies d'excitation électronique des gaz. Ce type de pompage n'est cependant pas idéal étant donné la grande étendue du spectre d'énergie des électrons créés soit dans les décharges (0,1-100 eV) soit au moyen de canons (100 keV-1 MeV). De ce point de vue les lasers constituent de bien meilleurs sources d'excitation. Il faut toutefois que la longueur d'onde d'émission soit appropriée.

Il apparaît aujourd'hui que les lasers UV moléculaires constituent un groupe bien particulier par rapport à l'ensemble des lasers. Cette différenciation est la conséquence des caractéristiques propres au milieu laser et aux types de transitions, ainsi qu'à leurs performances (domaine d'émission, énergie rayonnée, cohérence, durée d'émission) et à leurs applications. Deux familles se distinguent au sein de ce groupe, chacune d'elles étant caractérisée par des performances et des applications différentes liées à la technologie de pompage : la décharge pulsée dans le gaz, et l'excitation au moyen d'un faisceau d'électrons créés par canon. Ce dernier mode d'excitation a fourni jusqu'à présent les résultats les plus spectaculaires (densité d'énergie de l'ordre de $92 \mathrm{~J}$ à $1933 \AA$ à partir de $\mathrm{ArF}$ et $108 \mathrm{~J}$ à $2490 \AA$ à partir de $\mathrm{KrF}$ [10]). Cependant les moyens attachés à cette technologie sont très importants comparés à l'autre méthode. Celle-ci permet de créer des émissions moins énergétiques (d'un facteur $10^{3}$ environ) mais présente l'avantage d'être à l'échelle d'un nombre plus grand d'applications. C'est la raison pour laquelle nous avons choisi ici de ne traiter que du problème des lasers UV moléculaires à excitation électronique transverse par décharges. D'autre part ce type particulier de lasers offre encore un certain nombre de perspectives de développement qu'il convient de ne pas négliger dans la recherche de nouvelles transitions lasers.

\section{Systèmes moléculaires et mécanismes d'inversion.} - Contrairement aux espèces atomiques qui mettent souvent en jeu des matériaux ionisés, les transitions lasers moléculaires observées jusqu'à présent correspondent pour la plupart à des molécules neutres. (Le cas de la bande $4278 \AA$ du premier système négatif de $\mathrm{N}_{2}^{+}$sort du cadre de cette étude [11].) Il est vraisemblable que la principale raison soit que le temps de recombinaison des ions moléculaires est plus court que celui des ions atomiques.

Suivant la classification proposée par la physique moléculaire, les systèmes moléculaires lasers peuvent être rangés dans deux catégories, suivant le type de transition : celle-ci a lieu soit entre deux états liés, soit entre un état supérieur lié et un état inférieur peu lié ou répulsif. Bien que d'origine structurelle, cette distinction correspond à des lasers dont les caractéristiques d'émission sont sensiblement différentes. C'est la raison pour laquelle elle mérite d'être retenue. Le premier type correspond au schéma désormais classique suivant lequel les niveaux supérieur et inférieur de la transition représentent des configurations différentes de la molécule (Fig. 1a). Dans le cas du second type au contraire, le niveau inférieur laser est un continuum d'états d'énergie positifs qui décrit le mouvement libre d'une infinité de fragments du système de l'état supérieur lié (Fig. $1 b$ ). De ce fait, les largeurs de raie des transitions sont dans ce cas 


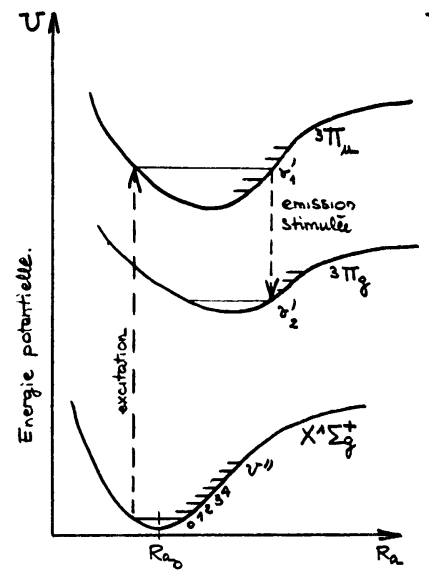

(a)

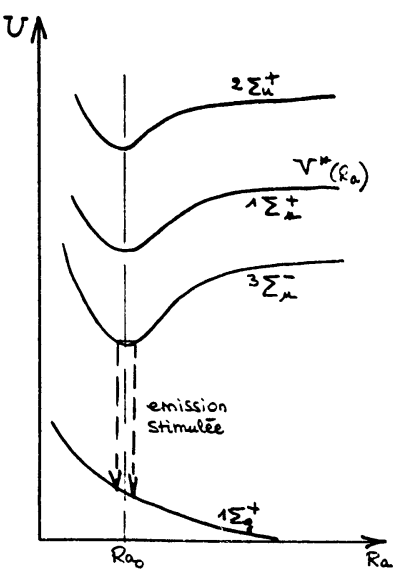

(b)
Fig. 1. - Schémas de principe d'une transition laser : $a$ ) entre états liés ; $b$ ) entre un état supérieur lié et un état répulsif.

supérieures de plusieurs ordres de grandeur à celles associées aux transitions entre états liés [12]. Etant donné ces différences de structures, les mécanismes d'inversion de population seront très différents. En particulier, dans le cas où des molécules du second type (excimères) ne peuvent pas exister à l'état libre (par exemple : $\mathrm{XeF}, \mathrm{KrF}, \mathrm{ArF}, \mathrm{XeCl}, \mathrm{XeBr}, \mathrm{KrCl}$, $\mathrm{ArCl}, \ldots)$ les processus de création de ces molécules dans l'état excité approprié joueront un rôle très important.

2.1 EMISSION LASER UV A PARTIR DE TRANSITIONS ENTRE ÉTATS ÉLECTRONIQUES LIÉS. - Des transitions lasers de ce type ont été observées à partir de quelques molécules diatomiques : $\mathrm{N}_{2}, \mathrm{H}_{2}, \mathrm{D}_{2}, \mathrm{HD}$ et $\mathrm{CO}$. Leurs principales caractéristiques sont rappelées tableau I. Notons que l'observation de ces émissions à partir de la $4^{\mathrm{e}}$ bande positive de $\mathrm{CO}$ n'a été faite que par Hodgson [13]. La nature particulièrement

Tableau I. - Caractéristiques des principales émissions UV lasers observées à partir de transitions entre états liés.

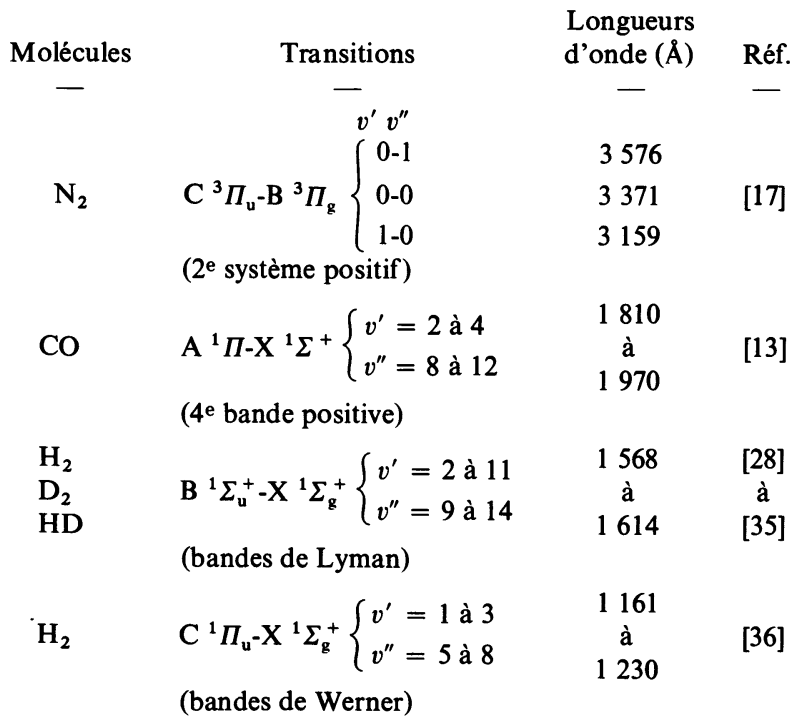

toxique de ce gaz a certainement constitué un frein à l'expérimentation de ce système. Egalement, les émissions observées n'ont pas semblé très intenses. D'autre part, les molécules $D_{2}$ et HD présentent de grandes similitudes de structures avec la molécule $\mathrm{H}_{2}$, aussi les résultats obtenus dans leur cas seront-ils regroupés avec ceux de $\mathrm{H}_{2}$. De ce fait, l'analyse de ce type de transition laser pourra être illustrée par les deux principales molécules du groupe : $\mathrm{N}_{2}$ et $\mathrm{H}_{2}$.

$\mathrm{Si}$ Heard [14] fut historiquement le premier à observer une émission stimulée à $3371 \AA$ à partir de $\mathrm{N}_{2}$, c'est en fait à Shipman [15] que l'on doit le développement de l'ensemble de la classe de lasers que nous étudions ici. L'apport de la technologie a été décisif. Bien que l'habitude ait conduit à ne retenir qu'une seule fréquence d'émission stimulée à partir de $\mathrm{N}_{2}$, plusieurs émissions sont en fait observées couramment. Elles correspondent toutes au second système positif $\mathrm{C}^{3} \Pi_{\mathrm{u}}-\mathrm{B}^{3} \Pi_{\mathrm{g}}$ et leurs conditions d'apparition ne sont pas affectées par celle du signal laser IR qui correspond à la transition $\mathrm{B}^{3} \Pi_{\mathrm{g}}-\mathrm{A}^{3} \Pi_{\mathrm{g}}[16]$ (Fig. 2).

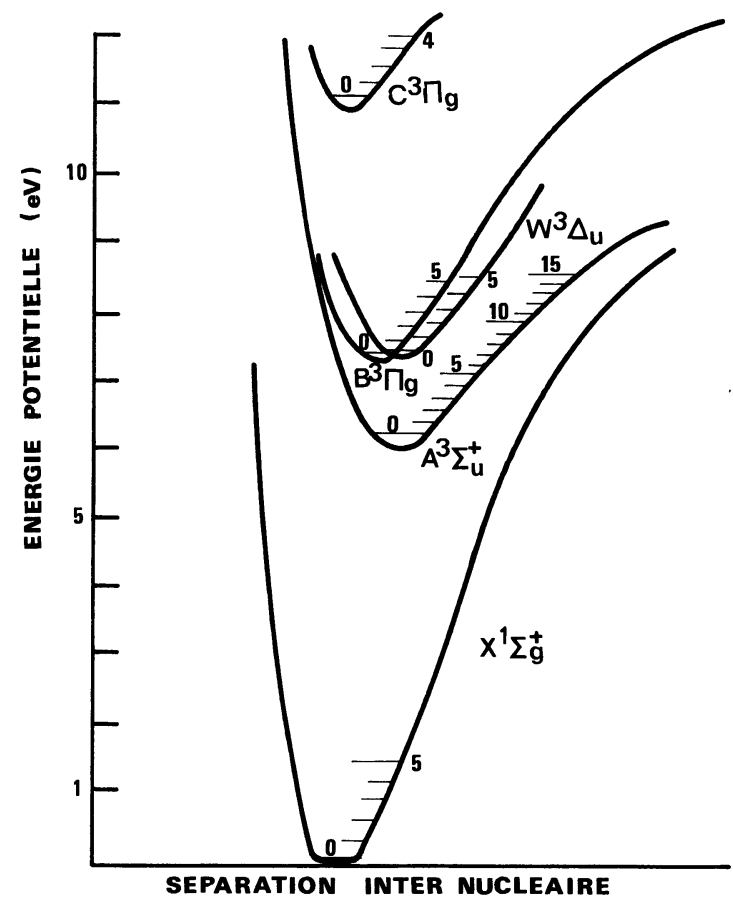

Fig. 2. - Courbes d'énergie potentielle de la molécule $\mathrm{N}_{2}$.

Toutes les observations confirment cependant la prédominance de la bande $\mathrm{C}^{3} \Pi\left(v^{\prime}=0\right)$-B ${ }^{3} \Pi\left(v^{\prime \prime}=0\right)$ dont la tête correspond à $\lambda=3371 \AA$. L'apparition des bandes (0-1), (1-0), (0-2) et (2-0) dépend des probabilités de transition estimées à partir des facteurs de Franck-Condon (Tableau II), mais également de la température du gaz et des qualités du système de pompage. Du point de vue des durées de vie des niveaux inférieurs et supérieurs lasers, cette transition présente une singularité défavorable à priori à l'émission stimulée. Elles sont en effet de l'ordre de

$\tau_{\mathrm{C}} \simeq 4 \times 10^{-8} \mathrm{~s}$ et $\tau_{\mathrm{B}} \simeq 10^{-5} \mathrm{~s}$. 
Tableau II. - Probabilités de transition dans le $2 \mathrm{e}$ système positif de $\mathrm{N}_{2}$.

$\begin{array}{cccc}\mathrm{C}^{3} \Pi_{\mathrm{u}}\left(v^{\prime}\right) & \mathrm{B}{ }^{3} \Pi_{\mathrm{g}}\left(v^{\prime \prime}\right) & (\mathrm{nm}) & \text { Probabilité } \\ \overline{0} & \overline{0} & - & - \\ 0 & 1 & 337,1 & 0,254 \\ 1 & 0 & 357,7 & 0,185 \\ 0 & 2 & 315,9 & 0,117 \\ 2 & 0 & 297,7 & 0,081 \\ & & & 0,014\end{array}$

On remarque que la durée de vie du niveau inférieur est très grande comparée à celle du niveau supérieur. C'est un exemple de transition dite auto-bloquée. La principale conséquence est l'impossibilité d'obtenir une émission stimulée continue à partir de cette transition. De plus, un calcul simple [17] montre que la durée d'émission stimulée ne peut excéder la durée de vie du niveau supérieur laser. Pour être efficace, le mode d'excitation de la molécule doit tenir compte de cette donnée fondamentale et doit être pulsé, relativement bref et son temps de montée ne doit pas excéder 10 ns environ. Cette condition n'est pas suffisante. Le pompage doit également tenir compte des seuils d'excitation des niveaux $B$ et $C$ et du seuil d'ionisation de la molécule $\mathrm{N}_{2}$. Ceux-ci sont respectivement de l'ordre de $E_{\mathrm{B}} \sim 7,4 \mathrm{eV}, E_{\mathrm{C}} \sim 11 \mathrm{eV}$ [18] et $E_{\mathrm{i}} \sim 15,6 \mathrm{eV}$ [19]. Comme nous l'avons déjà mentionné, le spectre d'énergie des électrons créés dans une décharge électrique est très étendu $[0,1-100 \mathrm{eV}]$. Dans ces conditions une part non négligeable des électrons ne peut participer directement au peuplement du niveau C. Soit leur énergie est trop faible, soit ils ionisent préalablement les molécules $\mathrm{N}_{2}$ et provoquent ainsi la création d'électrons secondaires. Malgré un taux de recombinaison élevé des électrons avec les molécules $\mathrm{N}_{2}^{+}$, on peut considérer que ces électrons participent momentanément à l'augmentation de la densité de courant dans le gaz et entraînent une chute rapide de la résistance du plasma ainsi créé [20]. L'efficacité du pompage tient compte simultanément des sections efficaces d'excitation de la molécule

$$
\left(\sigma_{\mathrm{B}} \sim 5 \times 10^{-17} \mathrm{~cm}^{2} \text { et } \sigma_{\mathrm{C}} \sim 7 \times 10^{-17} \mathrm{~cm}^{2}\right)
$$

L'observation d'émissions stimulées à partir de plusieurs bandes du $2^{\mathrm{e}}$ système positif, confirme l'hypothèse d'une excitation électronique directe de la molécule à partir de son état fondamental $\mathrm{X}^{1} \Sigma_{\mathrm{g}}^{+}$. Suivant le principe établi par Franck et Condon, le processus d'inversion de population s'appuie sur le décalage des minima des courbes d'énergie potentielle des niveaux $\mathrm{B}$ et $\mathrm{C}$ par rapport à $\mathrm{X}$, ainsi que sur les différences qui existent entre les sections efficaces d'excitation et de désexcitation des états électroniques mis en jeu.

L'analyse plus fine du spectre d'émission laser [22] pour chacune des trois bandes les plus intenses, met en évidence l'émission stimulée à partir d'un très grand nombre de niveaux rotationnels. L'apparition de cette structure fine rotationnelle est fortement liée à la pression totale d'azote. Ainsi lorsque cette pression croît de quelques torrs à quelques centaines de torrs, on observe une très sensible réduction du spectre laser. Les principales causes sont : l'élargissement des raies rotationnelles dans la région des têtes de branches $\mathbf{P}$ et $\mathbf{P}^{\prime}$, ainsi que l'accroissement de la constante de temps de relaxation rotationnelle par rapport à la durée de vie de la transition. La température initiale du gaz influe aussi sur le gain laser (qui croît lorsque la température décroît) ainsi que sur le déplacement du maximum relativement aux transitions rotationnelles. L'étude de l'évolution de ce gain, calculé à partir des équations de taux du système [23], en fonction du rapport du temps de montée $T$ de l'excitation sur la durée de vie $\tau_{\mathrm{C}}$ du niveau supérieur laser, montre que pour $T / \tau_{\mathrm{C}} \leqslant 1$, le gain varie peu et est maximum, tandis que pour $T / \tau_{\mathrm{C}}>1$, celui-ci décroît très rapidement. Ce résultat est lié à la nature auto-bloquée de la transition.

Une autre caractéristique importante de cette transition est la section efficace d'émission stimulée. Celle-ci est élevée, pour la raie $3371 \AA$, et a été estimée à $5 \times 10^{-17} \mathrm{~cm}^{2}$ [24], ce qui confère au milieu un caractère super-radiant (confirmé par toutes les expériences). Une conséquence importante est qu'il n'est pas nécessaire de coupler le matériau amplificateur avec une cavité résonnante. Ceci n'a pas cependant que des avantages, car la cohérence spatiale de ce type de laser est peu élevée comparativement à celle obtenue avec d'autres types de lasers tels que : $\mathrm{He}-\mathrm{Ne}$, rubis, $\mathrm{Nd}$ ou $\mathrm{CO}_{2}$. Egalement, ce caractère limite les possibilités de stockage d'énergie dans le matériau et réduit de ce fait la densité d'énergie rayonnante extraite. L'expérience montre que le rendement de ce type de lasers est toujours inférieur à $1 \%$ et l'accroissement de la puissance rayonnée se traduit partiellement par une réduction de la durée d'émission. L'étude du système montre d'autre part que malgré la valeur du gain, l'amplification de la lumière au cours d'un seul passage à travers le matériau n'entraîne pas une totale réduction de l'inversion de population. Ceci est démontré par la très sensible augmentation de l'énergie totale rayonnée lorsqu'un miroir de réflexion totale est placé à l'une des extrémités du milieu (ce facteur est d'autant plus élevé que la longueur d'amplification est petite).

Il a été montré par ailleurs que l'addition de $\mathrm{SF}_{6}$ à $\mathrm{N}_{2}$ entraîne une augmentation de l'énergie extraite du milieu (environ un facteur 2) [25]. Cette addition provoque également une forte augmentation de l'inversion de population dans la bande (0-1) autour de $3577 \AA$. Trois hypothèses peuvent être avancées : il y a désactivation des niveaux $\mathrm{B}^{3} \Pi_{\mathrm{g}}$ et $\mathrm{A}^{3} \Pi_{\mathrm{g}}$ à la suite des collisions entre $\mathrm{SF}_{6}$ et $\mathrm{N}_{2}$, ces collisions ayant pour effet de prolonger la durée de l'émission; il y a transfert résonnant d'énergie entre $\mathrm{SF}_{6}$ et $\mathrm{N}_{2}$; une recombinaison des ions $\mathrm{N}_{2}^{+}+\mathrm{SF}_{6}^{-}$pourrait favoriser 
au moins au début de la décharge la densité d'inversion entre $\mathrm{C}\left(v^{\prime}=0\right)$ et $\mathrm{B}\left(v^{\prime \prime}=1\right)$. Cette inversion serait ensuite détruite par l'excitation directe du niveau $\mathrm{B}\left(v^{\prime \prime}=0\right)$ si l'on s'en tient aux valeurs des facteurs de Franck-Condon. Il faut noter que l'inconvénient majeur de cette addition est la pollution du milieu actif, consécutive à l'apparition de soufre.

Les premières suggestions sur la possibilité d'obtenir des émissions cohérentes dans l'UV du vide, entre $1000 \AA$ et $2000 \AA$, à partir de la molécule $\mathrm{H}_{2}$, furent émises par Bazhulin et coll. [26]. Il se trouve en effet qu'à la température ambiante, seuls les niveaux $\left(v^{\prime \prime}=0, J^{\prime \prime}=0,1\right)$ du niveau fondamental $\mathrm{X}^{1} \Sigma_{\mathrm{g}}^{+}$ de la molécule sont peuplés de manière significative, ceci à cause du grand espacement $(\simeq 0,5 \mathrm{eV})$ en les niveaux vibrationnels [12]. D'autre part, l'existence de deux niveaux électroniques excités de la molécule B ${ }^{1} \Sigma_{\mathrm{u}}^{+}$et $\mathrm{C}^{1} \Pi_{\mathrm{u}}$ dont les minima d'énergie potentielle sont déplacés vers les grandes valeurs de la distance internucléaire, suggère qu'une inversion de population peut être créée entre l'un de ces deux états excités et l'état fondamental X (Fig. 3). Les études de fluores-

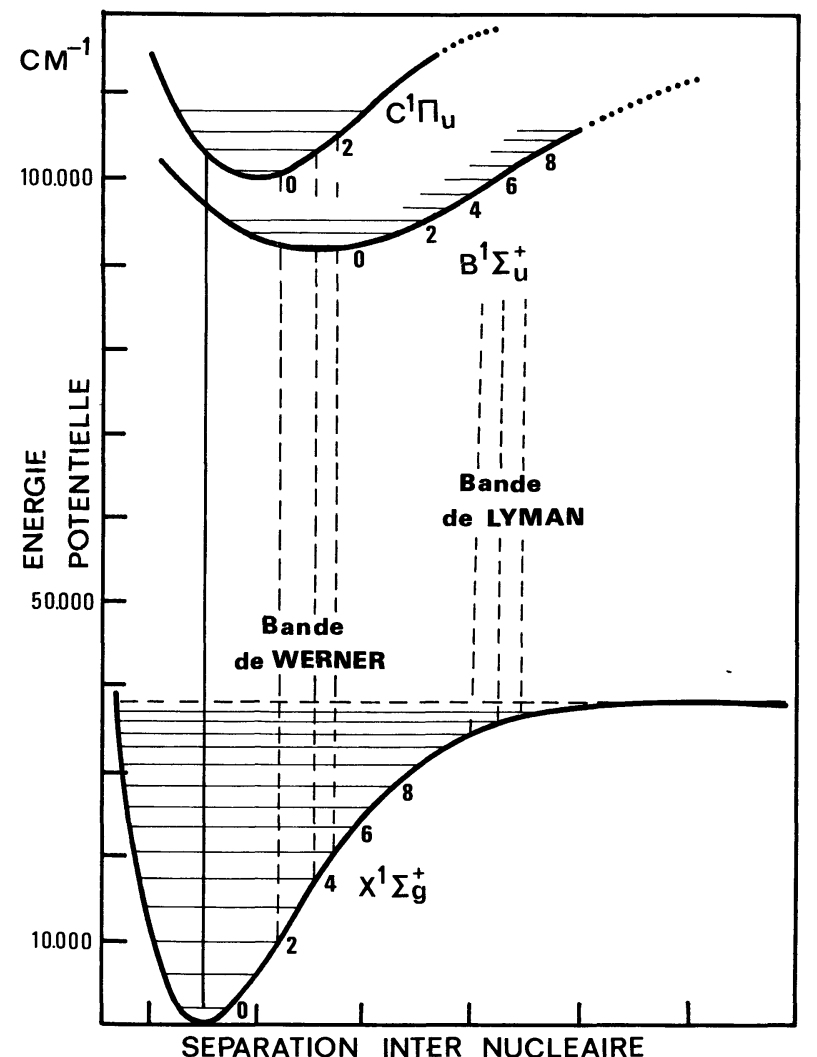

Fig. 3. - Courbes d'énergie potentielle de la molécule $\mathrm{H}_{2}$.

cence de la molécule $\mathrm{H}_{2}$ [27] montrent que la probabilité d'excitation est proportionnelle aux facteurs de Franck-Condon qui lient le niveau $\mathrm{X}^{1} \Sigma_{\mathrm{g}}^{+}\left(v^{\prime \prime}=0\right)$ avec les niveaux vibrationnels $v^{\prime}$ des états B et C. On remarque en particulier que ces facteurs sont très élevés pour les transitions

$\mathrm{B}\left(v^{\prime}=2,3,4,5,6,7\right)-\mathrm{X}\left(v^{\prime \prime}=9,10,11,12,13\right)$ et $\mathrm{C}\left(v^{\prime}=1,2,3,4\right)-\mathrm{X}\left(v^{\prime \prime}=4,5,6,7,8\right)$. Les niveaux $v^{\prime \prime} \neq 0$ étant quasiment vides et n'étant pas peuplés de manière appréciable lors de la décharge, ces données suggèrent qu'une inversion de population entre ces niveaux est possible. Les processus d'excitation et de désexcitation les plus importants sont : les collisions élastiques des électrons avec le noyau, l'excitation rotationnelle et vibrationnelle, l'excitation des niveaux singulets de la molécule, la dissociation et l'ionisation de la molécule ainsi que la création d'électrons secondaires. On note en particulier que les taux de relaxation et de prédissociation ont des ordres de grandeur compatibles avec l'inversion. Dans ces conditions, le rendement maximum d'excitation peut être obtenu pour une énergie moyenne des électrons comprise entre 10 et $20 \mathrm{eV}$ [28]. En tenant compte du fait que les durées de vie des transitions lasers sont inférieures à 1 ns, l'énergie électronique de $10 \mathrm{eV}$ ne sera obtenue que pour

$E / p \gtrsim 500 \mathrm{~V} \mathrm{~cm}^{-1} \times$ torr $^{-1}$,

ce qui est très élevé et plus facilement accessible dans le cas d'un pompage par canon.

Les expériences confirment cette analyse qui peut être également étendue aux molécules $D_{2}$ et $H D$. Plusieurs émissions lasers ont ainsi été observées (Tableau III) autour de $1600 \AA$ [29 à 34] et de $1200 \AA$ $[35,36]$. D'autres transitions ont aussi été prédites par Knyazev et coll. [28] dans les cas de $\mathrm{H}_{2}$ et $\mathrm{D}_{2}$ (Tableau IV), mais aucune confirmation expérimentale n'a encore été apportée. Au contraire de $\mathrm{N}_{2}$, l'hydrogène se caractérise par un spectre laser beaucoup

Tableau III. - Bandes lasers de $\mathrm{B}^{1} \Sigma_{\mathrm{u}}^{+}-\mathrm{X}^{1} \Sigma_{\mathrm{g}}^{+}$de $\mathrm{H}_{2}$.

\begin{tabular}{|c|c|c|c|}
\hline$v^{\prime}-v^{\prime \prime}$ & Raies & $\begin{array}{l}\text { Longueurs } \\
\text { d'onde }(\AA)\end{array}$ & Références \\
\hline- & $\overline{\mathrm{P} 1}$ & - & $028 \overline{-}$ \\
\hline $2-9$ & $\left\{\begin{array}{l}\mathrm{P} 1 \\
\mathrm{P} 2 \\
\mathrm{P} 3\end{array}\right.$ & $\begin{array}{l}1571,8 \\
1574,3 \\
1577,0\end{array}$ & $\begin{array}{l}{[28,30]} \\
{[28]} \\
{[28]}\end{array}$ \\
\hline $3-10$ & $\left\{\begin{array}{l}\text { P1 } \\
\text { P3 }\end{array}\right.$ & $\begin{array}{l}1591,5 \\
1596,1\end{array}$ & $\begin{array}{l}{[30]} \\
{[29,30,34]}\end{array}$ \\
\hline $4-11$ & $\left\{\begin{array}{l}\text { P1 } \\
\text { P3 }\end{array}\right.$ & $\begin{array}{l}1604,5 \\
1608,4\end{array}$ & $\begin{array}{l}{[28,29,30]} \\
{[28,29,30,34]}\end{array}$ \\
\hline $5-12$ & $\left\{\begin{array}{l}\text { P1 } \\
\text { P2 } \\
\text { P3 } \\
\text { P4 } \\
\text { P5 }\end{array}\right.$ & $\begin{array}{l}1610,3 \\
1611,6 \\
1613,2 \\
1614,8 \\
1616,5\end{array}$ & $\begin{array}{l}{[28,29,30,34]} \\
{[29]} \\
{[28,29,30,34]} \\
{[28]} \\
{[28]}\end{array}$ \\
\hline $6-13$ & $\left\{\begin{array}{l}\text { P1 } \\
\text { P2 } \\
\text { P3 }\end{array}\right.$ & $\begin{array}{l}1607,5 \\
1608,3 \\
1609\end{array}$ & $\begin{array}{l}{[28,29,30,34]} \\
{[34]} \\
{[29]}\end{array}$ \\
\hline $7-13$ & $\left\{\begin{array}{l}\mathrm{P} 3 \\
\mathrm{R} 1\end{array}\right.$ & $\begin{array}{l}1580,5 \\
1577,1\end{array}$ & $\begin{array}{l}{[28,29,30,34]} \\
{[30]}\end{array}$ \\
\hline $8-14$ & $\left\{\begin{array}{l}\text { P1 } \\
\text { R1 }\end{array}\right.$ & $\begin{array}{l}1567,3 \\
1565,5\end{array}$ & $\begin{array}{l}{[28,30]} \\
{[28]}\end{array}$ \\
\hline $11-14$ & P3 & 1492,2 & [28] \\
\hline
\end{tabular}


Tableau IV. - Autres bandes susceptibles d'être laser de $\mathrm{H}_{2}$ et $\mathrm{D}_{2}$, d'après [28].

\begin{tabular}{|c|c|c|c|}
\hline \multirow{5}{*}{ Molécules } & $v^{\prime}-v^{\prime \prime}$ & Raies & $\begin{array}{l}\text { Longueur } \\
\text { d'onde }(\AA\end{array}$ \\
\hline & - & - & - \\
\hline & & P2 & 1567,525 \\
\hline & $8-14$ & P3 & 1567,249 \\
\hline & & P4 & 1566,431 \\
\hline \multirow{7}{*}{$\begin{array}{c}\mathrm{H}_{2} \\
(\mathrm{~B}-\mathrm{X})\end{array}$} & & $\mathrm{P} 2$ & 1518,184 \\
\hline & $10-14$ & P3 & 1518,005 \\
\hline & & P4 & 1517,343 \\
\hline & & P2 & 1495,686 \\
\hline & & P3 & 1495,565 \\
\hline & $11-14$ & P4 & 1495,012 \\
\hline & & P5 & 1494,190 \\
\hline \multirow{4}{*}{$\begin{array}{c}\mathrm{D}_{2} \\
(\mathrm{~B}-\mathrm{X})\end{array}$} & & P2 & 1596,249 \\
\hline & $11-20$ & P3 & 1596,115 \\
\hline & & P4 & 1595,772 \\
\hline & $12-21$ & P1 & 1580,193 \\
\hline
\end{tabular}

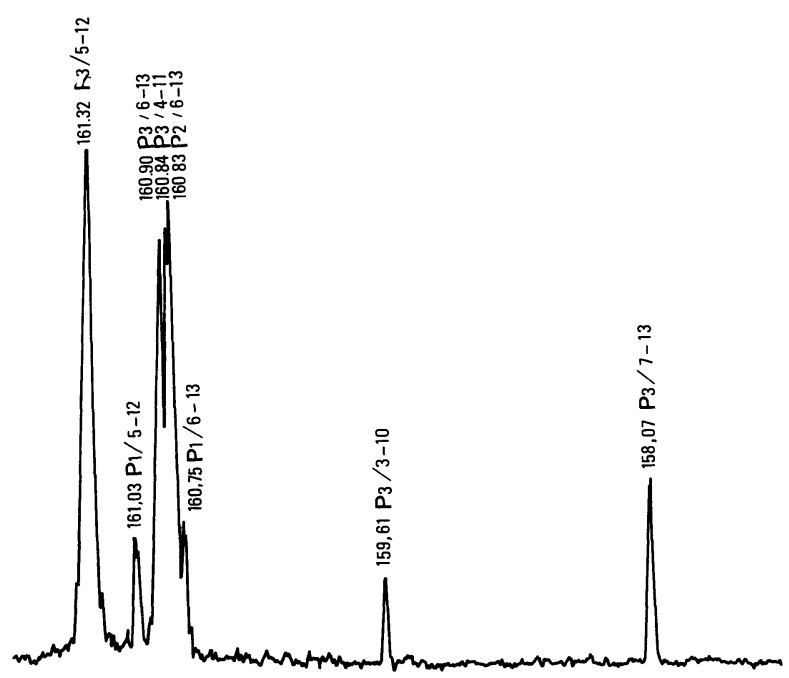

Fig. 4. - Densitogramme du spectre d'émission stimulée à partir de la bande de Lyman de $\mathrm{H}_{2}$ [34]. plus dense (Fig. 4). Enfin, l'analyse spectrale de cette émission fait apparaître une réduction importante du nombre des raies lasers lorsque la pression d'hydrogène devient supérieure à 100 torrs environ. On observe également des rendements totaux d'émission inférieurs à ceux obtenus dans $\mathrm{N}_{2}$, qui sont de l'ordre de $10^{-5}$, et qui dépendent là aussi des caractéristiques du montage expérimental.

2.2 TRansitions ENTRE UN ÉTAT LIÉ ET UN ÉTAT DissoCiATIF. - C'est en 1960 que Houtermans [37] montra l'intérêt qu'il y aurait à rechercher des transitions entre un état supérieur lié et un état inférieur dissociatif pour obtenir un effet laser. L'avantage dans ce cas est que l'instabilité de l'état électronique inférieur, qui se traduit par la dissociation de la molécule en deux atomes répulsifs, conduit en théorie à disposer d'un niveau inférieur toujours vide. Cette situation permet d'envisager la création d'une inversion de population assez facilement et dans le cas où cette inversion peut être maintenue, il est possible d'espérer avoir une émission stimulée continue. La première vérification expérimentale a été faite par Basov [38] en 1970 à partir du xénon liquide. Depuis cette date, un certain nombre de molécules diatomiques présentant cette particularité de structure ont fourni plusieurs émissions stimulées dans le proche UV et l'UV du vide : $\mathrm{Xe}_{2}, \mathrm{Kr}_{2}, \mathrm{Ar}_{2}, \mathrm{~F}_{2}, \mathrm{Cl}_{2}$, $\mathrm{Br}_{2}, \mathrm{XeF}, \mathrm{KrF}, \mathrm{ArF}, \mathrm{XeCl}, \mathrm{KrCl}, \mathrm{ArCl}, \mathrm{XeBr}$. Les caractéristiques des transitions observées à partir de ces molécules excitées au moyen d'une décharge transverse sont reproduites tableau V. Comme le montre la liste ci-dessus, un beaucoup plus grand nombre de raies ont été observées à partir d'un pompage par faisceau d'électrons. Toutefois, ces émissions sont certainement à l'origine d'un renouveau du développement de la technologie de pompage par décharge.

L'analyse des transitions montre d'autre part que deux situations sont possibles : Dans le premier cas, l'état fondamental dissociatif de la molécule constitue

Tableau V. - Caractéristiques des principales émissions lasers obtenues à partir de molécules excimères excitées par une décharge.

\begin{tabular}{|c|c|c|c|}
\hline Molécules & Transitions & Mélanges initiaux & $\begin{array}{l}\text { Longueurs } \\
\text { d'onde } \\
\text { d'émission } \\
\text { stimulée }(\AA)\end{array}$ \\
\hline - & - & - & $\overline{3490}$ \\
\hline $\mathrm{XeF}$ & $\mathrm{C}^{2} \Sigma_{1 / 2}^{+}-\mathrm{X}^{2} \Sigma_{1 / 2}^{+}$ & Ar ou $\mathrm{He}$ ou $\mathrm{Ne}: \mathrm{Xe}: \mathrm{F}_{2}$ ou $\mathrm{NF}_{3}$ & $\begin{cases}3 & 510 \\
3 & 530\end{cases}$ \\
\hline $\mathrm{XeCl}$ & $\mathrm{C}^{2} \Sigma_{1 / 2}^{+}-\mathrm{X}^{2} \Sigma_{1 / 2}^{+}$ & $\mathrm{He}: \mathrm{Xe}: \mathrm{Cl}_{2}$ ou $\mathrm{BCl}_{3}$ & 3076 à 3084 \\
\hline $\mathrm{KrF}$ & $\mathrm{C}^{2} \Sigma_{1 / 2}^{+}-\mathrm{X}^{2} \Sigma_{1 / 2}^{+}$ & Ar ou $\mathrm{He}: \mathrm{Kr}: \mathrm{F}_{2}$ ou $\mathrm{NF}_{3}$ ou $\mathrm{N}_{2} \mathrm{~F}_{4}$ & 2490 \\
\hline $\mathrm{KrCl}$ & $\Sigma_{1 / 2}^{+}-\Sigma_{1 / 2}^{+}$ & $\mathrm{Kr}: \mathrm{Cl}_{2}$ & 2229 \\
\hline $\mathrm{ArF}$ & $\mathrm{C}^{2} \Sigma_{1 / 2}^{+}-\mathrm{X}^{2} \Sigma_{1 / 2}^{+}$ & $\mathrm{He}: \mathrm{Ar}: \mathrm{F}_{2}$ & 1930 \\
\hline $\mathrm{ArCl}$ & $\Sigma_{1 / 2}^{+}-\Sigma_{1 / 2}^{+}$ & $\mathrm{He}: \mathrm{Ar}: \mathrm{Cl}_{2}$ & 1750 \\
\hline
\end{tabular}


le niveau inférieur laser. On rencontre cette situation avec les molécules excimères $\mathrm{XeF}, \mathrm{KrF}$ et $\mathrm{ArF}$ par exemple. Dans le second cas, au contraire, l'état inférieur dissociatif de la transition est un état excité de la molécule. Une telle situation est illustrée par $\mathrm{Cl}_{2}$ ou $\mathrm{Br}_{2}$. Nous porterons donc notre attention successivement sur chacune d'elles.

L'étude des molécules excimères montre que l'état excité est caractérisé par une fonction potentielle $V^{*}\left(R_{\mathrm{a}}\right)$ qui présente un minimum marqué pour une séparation internucléaire $R_{\mathrm{a} 0}$ et il existe pour cet état plusieurs niveaux de vibration. Au contraire le potentiel $V\left(R_{\mathrm{a}}\right)$ de l'état fondamental est soit peu lié, soit répulsif pour $R_{\mathrm{a}} \sim R_{\mathrm{a} 0}$, tandis qu'il présente un minimum peu marqué en général à une distance $R_{\mathrm{a}} \gg R_{\mathrm{a} 0}$ (Fig. 1b). Il faut aussi observer que l'état triplet ${ }^{3} \Sigma_{\mathrm{u}}^{+}$est placé juste au-dessous de l'état singulet ${ }^{1} \Sigma_{\mathrm{u}}^{+}$. Etant donné les caractéristiques de l'état fondamental, on observe une largeur de raie d'émission beaucoup plus importante que dans le cas d'une transition entre états liés. Notons enfin que certaines de ces bandes d'émission continues sont observées avec des caractéristiques voisines dans les trois phases : gaz, liquide et solide. Ceci explique que l'on ait d'abord recherché à obtenir des émissions à partir de matériaux liquides ou solides. Les gaz restent toutefois plus facilement accessibles.

Ces molécules présentent l'inconvénient de ne pas exister à l'état naturel et l'exploitation des avantages de ce type de transition rend préalablement nécessaire la création d'un nombre important de molécules dans l'état excité supérieur laser. Pour y parvenir, la seule voie consiste à faire réagir plusieurs atomes ou molécules entre eux. Pour illustrer ce propos, considérons le cas particulier de la formation de la molécule $\mathrm{XeF}^{*}$ à partir du mélange $\mathrm{Xe}+\mathrm{NF}_{3}$. Un certain nombre de réactions sont possibles dont :

$\mathrm{e}+\mathrm{Xe} \rightarrow \mathrm{Xe}^{*}+\mathrm{e}$

$\mathrm{Xe}^{*}+\mathrm{NF}_{3} \rightarrow \mathrm{XeF}^{*}+\mathrm{NF}_{2}$

$\mathrm{XeF}^{*} \rightarrow \mathrm{Xe}+\mathrm{F}+\mathrm{h} v(3510 \AA)$

la molécule de $\mathrm{NF}_{3}$ pouvant ensuite se reformer à partir de la réaction $\mathrm{NF}_{2}+\mathrm{F}$ par exemple. L'expérience indique cependant que le mélange initial tend à se dégrader après un certain nombre d'excitation [39]. Dans le cas d'un pompage transverse, on a remarqué d'autre part que l'émission stimulée n'apparaissait qu'à la condition de rajouter un gaz diluant au mélange initial. Les diluants couramment utilisés sont l'hélium, l'argon et le néon. L'argon semble favoriser la formation d'arcs et on lui préfère en général $\mathrm{He}$ ou Ne. Champagne [40] a montré dans le cas de XeF que l'emploi du néon était préférable à celui de l'argon lorsque la pression totale du mélange devient supérieure à 1,3 atm. Dans certains cas, par exemple $\mathrm{KrF}^{*}$, l'usage d'un diluant tel que l'argon peut entraîner la formation concurrente d'une autre molécule excimère :

$$
\begin{aligned}
& \mathrm{e}+(\mathrm{Kr}, \mathrm{Ar}) \rightarrow\left(\mathrm{Kr}^{*}, \mathrm{Ar}^{*}\right)+\mathrm{e} \\
& \left(\mathrm{Kr}^{*}, \mathrm{Ar}^{*}\right)+\mathrm{F}_{2} \rightarrow\left(\mathrm{KrF}^{*}, \mathrm{ArF}^{*}\right)+\mathrm{F} \\
& \mathrm{KrF}^{*} \rightarrow \mathrm{Kr}+\mathrm{F}+\mathrm{h} v \quad(2490 \AA) \\
& \mathrm{ArF}^{*} \rightarrow \mathrm{Ar}+\mathrm{F}+\mathrm{h} v \quad(1930 \AA) .
\end{aligned}
$$

D'autres processus de formation de la molécule excimère peuvent avoir lieu, qui tiennent compte par exemple du phénomène d'attachement dissociatif des électrons par l'halogène, suivi par une recombinaison ion-ion à trois corps :

$$
\begin{aligned}
\mathrm{e}+\mathrm{F}_{2} \rightarrow \mathrm{F}^{-}+\mathrm{F} \\
\mathrm{He}+\left(\mathrm{Xe}^{+}, \mathrm{Kr}^{+}, \mathrm{Ar}^{+}\right)+\mathrm{F}^{-} \rightarrow \\
\quad \rightarrow\left(\mathrm{XeF}^{*}, \mathrm{KrF}^{*}, \mathrm{ArF}^{*}\right)+\mathrm{He} .
\end{aligned}
$$

Pour estimer le rendement réel de tels systèmes, il faut tenir compte de tous les processus de pertes d'énergie qui réduisent de manière appréciable le gain laser et qui' sont inévitables étant donné le très grand nombre de réactions possibles. Les principaux processus cinétiques ainsi que l'ordre de grandeur des constantes de réaction $k$ ont été rassemblés dans le tableau VI. On note que dans le cas de réac-

Tableau VI. - Principaux processus cinétiques intervenant lors de la formation des molécules $\mathrm{XeF}^{*}, \mathrm{KrF}^{*}$ et $\mathrm{ArF}^{*}$, d'après les références [42], [78-82].

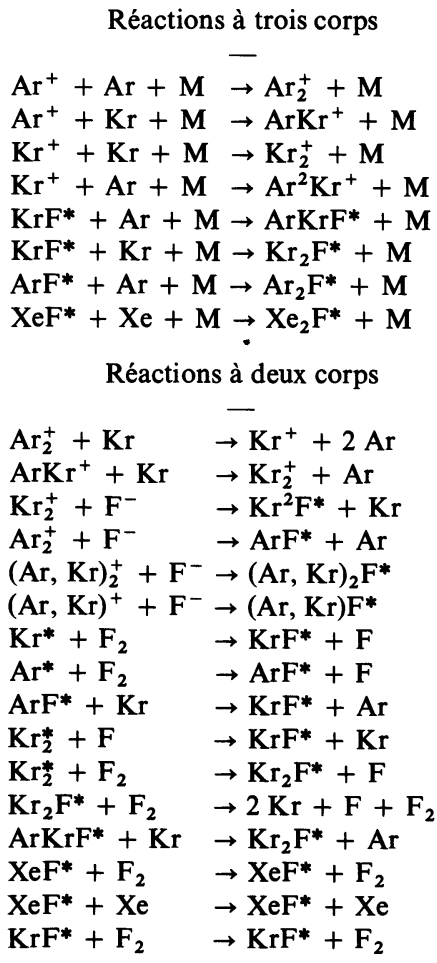

Réactions avec un photon

$\mathrm{KrF}^{*}+\mathrm{h} v \rightarrow \mathrm{Kr}+\mathrm{F}+\mathrm{h} v$

$\mathrm{F}_{2}+\mathrm{h} v \rightarrow \mathrm{F}+\mathrm{F}$

$\mathrm{F}^{-}+\mathrm{h} v \quad \rightarrow \mathrm{F}+\mathrm{e}$

$\mathrm{Ar}_{2}^{+}+\mathrm{h} v \rightarrow \mathrm{Ar}+\mathrm{Ar}^{+}$

$\mathrm{Kr}_{2}^{+}+\mathrm{h} v \quad \rightarrow \mathrm{Kr}+\mathrm{Kr}^{+}$

$$
\begin{array}{rl}
k\left(\mathrm{~cm}^{6} \mathrm{~s}^{-1}\right) & - \\
2 \mathrm{à} 2,5 & \times 10^{-31} \\
2,5 & \times 10^{-31} \\
2,4 \mathrm{à} & 2,5 \times 10^{-31} \\
1,0 & \times 10^{-31} \\
6 \mathrm{à} 9 & \times 10^{-32} \\
5 \mathrm{à} 6,5 & \times 10^{-31} \\
4 & \times 10^{-31} \\
7,8 & \times 10^{-31} \\
k\left(\mathrm{~cm}^{3} \mathrm{~s}^{-1}\right) & \\
7 & \\
7,5 & \times 10^{-10} \\
3,2 & \times 10^{-10} \\
2 \mathrm{à} 3 & \times 10^{-6} \\
5 & \times 10^{-7} \\
5 & \times 10^{-7} \\
1 & \times 10^{-6} \\
7,2 & \times 10^{-10} \\
7,5 & \times 10^{-10} \\
1,5 \mathrm{à} 5,5 & \times 10^{-10} \\
3 & \times 10^{-10} \\
3 & \times 10^{-10} \\
1 & \times 10^{-9} \\
1 & \times 10^{-10} \\
3 & \times 10^{-10} \\
2,9 & \times 10^{-10} \\
7,8 & \times 10^{-10} \\
k & \left(\mathrm{~cm}{ }^{2}\right) \\
- & \\
2 & \times 10^{-16} \\
1,5 & \times 10^{-20} \\
5,4 & \times 10^{-18} \\
1,5 & \times 10^{-17} \\
1 & \times 10^{-18}
\end{array}
$$


tions à trois corps, les constantes $k$ sont toutes du même ordre de grandeur et voisines de $10^{-31} \mathrm{~cm}^{6} \mathrm{~s}^{-1}$. $\mathrm{Au}$ contraire, les constantes $k$ de réaction des processus à deux corps varient de plusieurs ordres de grandeur, entre $10^{-6}$ et $10^{-10} \mathrm{~cm}^{3} \mathrm{~s}^{-1}$, indiquant par là que leurs rôles sont très différents suivant le mélange et la transition laser recherchée. D'autre part, les sections efficaces d'émission stimulée à partir de ces molécules sont très élevées $\left(\simeq 2 \times 10^{-16} \mathrm{~cm}^{2}\right.$ [43] pour la transition $2490 \AA$ de $\left.\mathrm{KrF}^{*}\right)$. Ceci permet d'espérer un gain laser également élevé, malgré les faibles durées de vie des transitions $(6,5$ ns dans $\mathrm{KrF}^{*}$ [44] et $10 \mathrm{~ns}$ dans $\mathrm{ArF}^{*}$ [45]). L'expérience confirme ce point de vue puisque des énergies supérieures à $100 \mathrm{~mJ}$ ont pu être extraites de $\mathrm{KrF}$ et $\mathrm{ArF}$ et de l'ordre de $250 \mathrm{~mJ}$ à partir de $\mathrm{XeF}$ avec des rendements supérieurs à $1 \%$.

La seconde situation, présentée au début de ce paragraphe, offre un incontestable avantage sur la première, dans la mesure où la transition laser prenant appui entre deux niveaux excités de la molécule, celle-ci peut exister naturellement dans son état fondamental. Cette situation peut être illustrée par le groupe des molécules hallogènes $\mathrm{Cl}_{2}, \mathrm{Br}_{2}, \mathrm{I}_{2}$ par exemple. Ainsi dans le cas de $I_{2}$, la transition laser correspond à la bande ${ }^{3} \Pi_{2 \mathrm{~g}} \rightarrow{ }^{3} \Pi_{2 \mathrm{u}}$ (Fig. 5). La démonstration d'une

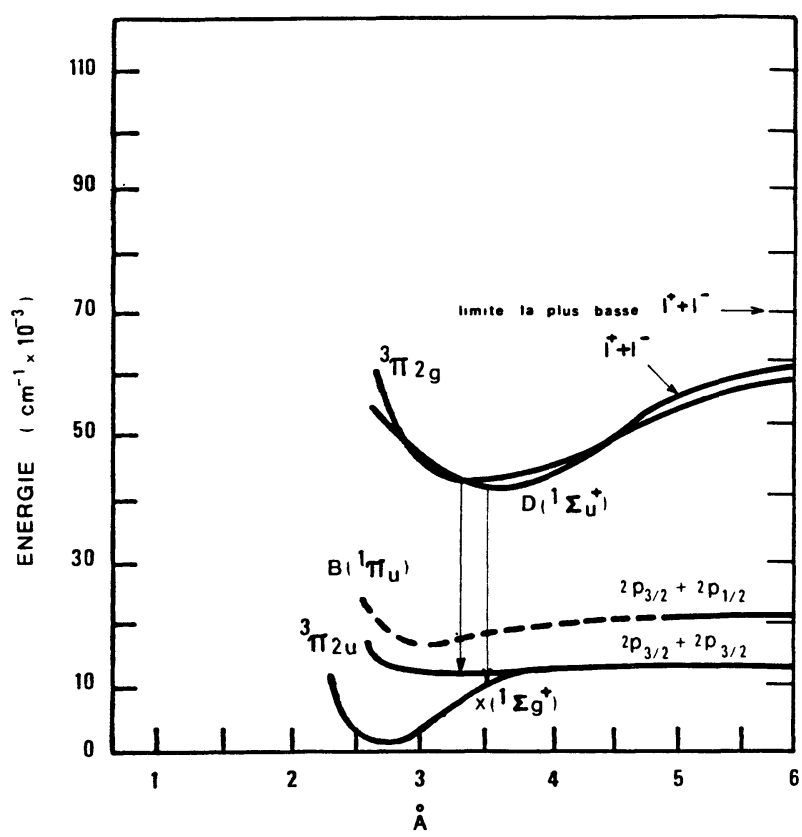

Fig. 5. - Courbes d'énergie potentielle de la molécule $I_{2}$ d'après les références [47] et [48].

émission stimulée à partir de cette bande, entre $3450 \AA ̊$ et $3040 \AA$, a été faite initialement par McCusker et coll. [46]. Mulliken suggère dans son article de synthèse sur la molécule $I_{2}$ [47] que certaines bandes de $D$-X pourraient également être présentes, mais l'analyse plus récente de Tellinghuisen [48] lève l'ambiguïté et précise que le maximum de densité d'énergie devrait pouvoir être extrait à partir des bandes $0-12,2-15,3-17,1-14$ et $0-13$ de la transition ${ }^{3} \Pi_{2 \mathrm{~g}} \rightarrow{ }^{3} \Pi_{2 \mathrm{u}}$, au voisinage de $3400 \AA$.

Puisque l'état ${ }^{3} \Pi_{2 \mathrm{u}}$ est situé à environ $10000 \mathrm{~cm}^{-1}$ au-dessus de l'état fondamental, il semblerait possible d'inverser les populations des états ${ }^{3} \Pi_{2 \mathrm{~g}}$ et ${ }^{3} \Pi_{2 \mathrm{u}}$ au moyen d'une excitation électronique directe de $\mathrm{I}_{2}$ ou par transfert entre une molécule de gaz rare excitée $R$ et $I_{2}$. L'expérience réalisée par A. K. Hays et coll. [49] montre que l'excitation électronique directe (par canon à électrons) de $\mathrm{I}_{2}$ ne conduit pas à une émission laser. Au contraire, l'excitation de mélanges du type R-MI, dans lesquels MI représente un composé iodé ( $\mathrm{HI}, \mathrm{CH}_{3} \mathrm{I}$ ou $\mathrm{CF}_{3} \mathrm{I}$ ) permet d'observer une émission stimulée autour de $3425 \AA$ [50 à 52]. On note aussi que l'émission laser apparaît peu de temps après le front de l'impulsion électronique (15 à $30 \mathrm{~ns})$. Ce résultat suggère que le mécanisme de formation de $\mathrm{I}_{2}\left({ }^{3} \Pi_{2 \mathrm{~g}}\right)$ met en jeu des réactions entre espèces chargées :

$\mathrm{I}^{-}+\mathrm{I}^{+}+\mathrm{R} \rightarrow \mathrm{I}_{2}^{*}+\mathrm{R}$
$\mathrm{I}^{-}+\mathrm{MI}^{+}+\mathrm{R} \rightarrow \mathrm{I}_{2}^{*}+\mathrm{M}+\mathrm{R}$.

Le taux de recombinaison à 3 corps étant de l'ordre de $10^{-26} \mathrm{~cm}^{6} \mathrm{~s}^{-1}$ [53], $\mathrm{I}^{+}$et $\mathrm{MI}^{+}$pouvant être formés par différentes voies : collision des molécules MI avec des électrons de haute énergie, transfert de charge à partir de $\mathrm{R}^{+}$ou $\mathrm{R}_{2}^{+}$, transfert de charge dissociatif à

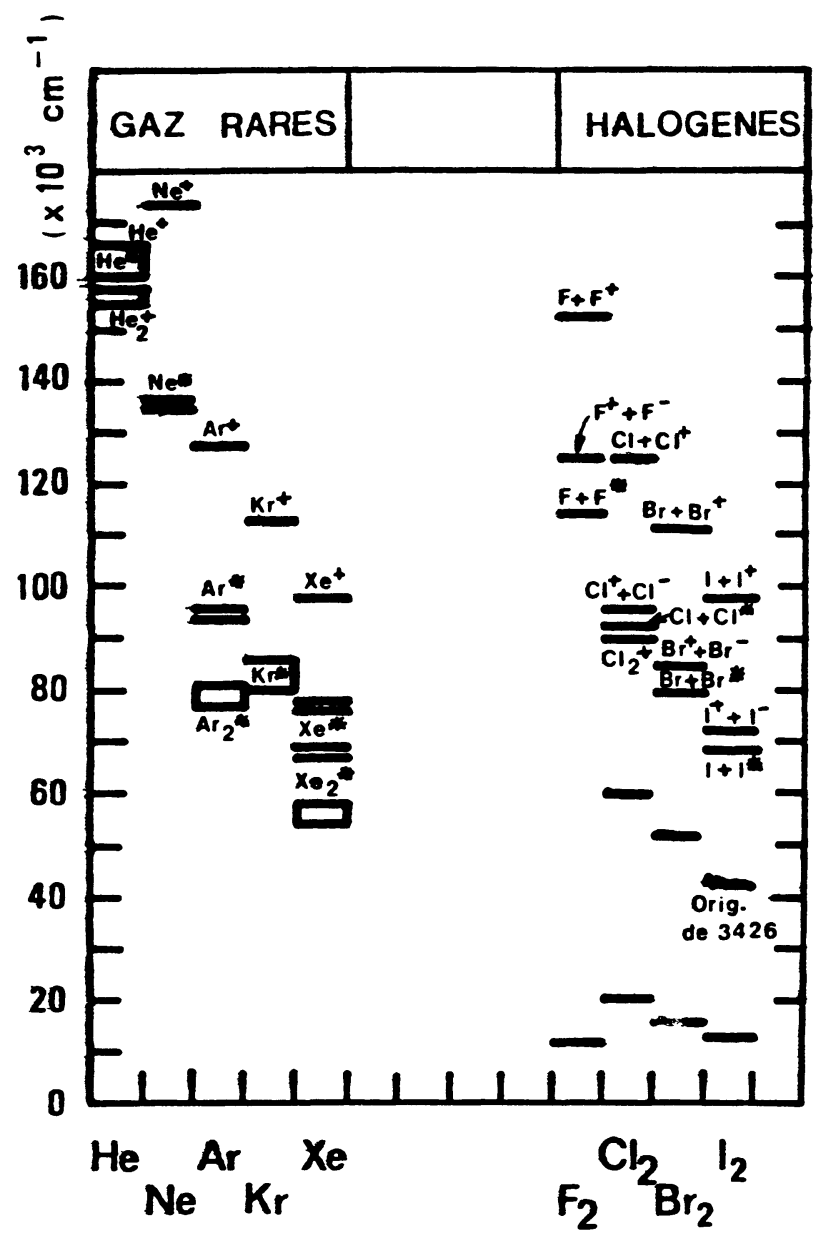

Fig. 6. - Niveaux d'énergie comparés des états excités de $\mathrm{He}, \mathrm{Ne}$, $\mathrm{Ar}, \mathrm{Kr}, \mathrm{Xe}$ et $\mathrm{F}_{2}, \mathrm{Cl}_{2}, \mathrm{Br}_{2}, \mathrm{I}_{2}$. 
partir de $\mathrm{R}^{+}$ou $\mathrm{R}_{2}^{+}$, ionisation de Penning de MI par $R_{2}^{+}$. Malgré les résultats expérimentaux actuels, rien ne s'oppose à priori à obtenir une émission laser au moyen d'un pompage transverse $(0,1-100 \mathrm{eV})$. Les niveaux d'énergie des atomes $\mathrm{Ar}$, $\mathrm{He}$ et $\mathrm{Ne}$ (Fig. 6) permettent d'envisager indifféremment leur emploi comme diluant. Toutefois si l'on se réfère à l'expérience acquise, l'emploi de $\mathrm{He}$ ou $\mathrm{Ne}$ devrait mieux convenir dans le cas d'une excitation par décharge.

La situation de $\mathrm{Br}_{2}$ est très similaire à celle de $\mathrm{I}_{2}$. Une première émission laser est observée à partir de la transition $\mathrm{B}^{3} \Pi_{\mathrm{u}}^{+} \rightarrow \mathrm{X}^{3} \Sigma_{\mathrm{g}}^{+}$[54] analogue à la transition visible $\mathrm{B}-\mathrm{X}$ de $\mathrm{I}_{2}$. La transition a lieu dans ce cas entre deux états liés de la molécule et les schémas d'inversion et d'émission sont du même type que ceux rencontrés pour $\mathrm{H}_{2}$. L'intérêt de ce système de bandes est qu'il est très étendu et susceptible de laser depuis $3500 \AA$ jusqu'à $5000 \AA$. La seconde transition laser correspond à $\mathrm{A}^{3} \Pi_{2 \mathrm{u}} \rightarrow{ }^{3} \Pi_{2 \mathrm{~g}}$, référence faite à l'analyse la plus récente [55]. Une émission stimulée a été observée au voisinage de $2920 \AA$ à partir de ce système [56-58] et Tellinghuisen a montré que cette émission s'établissait précisément entre $2913 \AA$ et $2926 \AA$, les bandes les plus intenses étant les bandes $(0-10)$, $(0-11),(1-12),(2-13)$ et (2-14). Le décalage entre cette transition et son homologue de $\mathrm{I}_{2}$ est d'environ $500 \AA$ vers les courtes longueurs d'onde. Il a été remarqué d'autre part que dans le cas où le diluant est Ar, la réaction $\left(\mathrm{Ar}^{*}+\mathrm{Br}_{2}^{*}\right)$ produisait peu de molécules d'halogénures de gaz rares tels que $\mathrm{ArBr}^{*}$ ou $\mathrm{ArBr}_{2}^{*}$, mais conduisait à la production d'états excités très élevés de $\mathrm{Br}(\simeq 98 \%)$. La formation de $\mathrm{Br}_{2}^{*}$ pourrait alors provenir de réactions du type [58] :

$\mathrm{Br}^{*}+\mathrm{Br}^{*} \rightarrow \mathrm{Br}_{2}^{*}$.

Etant donné le faible potentiel d'ionisation de $\mathrm{Br}^{*}$, celui-ci pourrait réagir avec $\mathrm{Br}_{2}$ pour former des produits ionisés :

$\mathrm{Br}^{*}+\mathrm{Br}_{2} \rightarrow \mathrm{Br}^{+} \mathrm{Br}^{-}+\mathrm{Br}$.

Les sections efficaces de ce type de réaction devraient être grandes et les états ionisés ainsi formés devraient transférer leur énergie lors de collisions avec $\mathrm{Br}_{2}$ qui pourrait être excité ainsi jusqu'à l'état supérieur laser. Cependant, tous ces phénomènes cinétiques sont encore trop peu connus pour permettre de faire une analyse définitive des phénomènes. Notons encore que le rendement de ce laser a été estimé à 10 ou $20 \%$ [59], ce qui accroîtrait son intérêt si cette valeur était confirmée.

Enfin, le cas de $\mathrm{Cl}_{2}$ est voisin de celui des deux précédentes molécules. Une émission laser est possible vers $2580 \AA$ à partir de la transition ${ }^{3} \Pi_{\mathrm{g}} \rightarrow{ }^{3} \Pi_{\mathrm{u}}[59]$. L'étude de la photodissociation de la molécule $\mathrm{OCCl}_{2}$ dans l'UV du vide, montre la production de molécules $\mathrm{Cl}_{2}$ dans plusieurs états excités [60]. En particulier, trois bandes centrées sur $2460 \AA, 2580 \AA$ et $3063 \AA$ sont observées. L'addition d'un gaz inerte tel que Ar entraîne l'extinction des bandes centrées sur $2460 \AA$ et $3063 \AA$, tandis qu'est amplifiée l'émission à $2580 \AA$. Ceci ne saurait constituer qu'une information, mais indique cependant qu'il serait intéressant de poursuivre l'étude de cette transition afin d'évaluer les possibilités d'émission laser.

3. Technologie et performances des lasers à excitation électronique par décharges transverses. - En dehors des cas très particuliers de $\mathrm{H}_{2}, \mathrm{HD}$ et $\mathrm{D}_{2}$, les durées de vie radiatives des molécules lasers dans l'ultraviolet sont de l'ordre d'une dizaine de nanosecondes au moins. Le temps de montée de l'excitation devra donc être du même ordre de grandeur pour être efficace. D'autres conditions doivent être satisfaites simultanément. Le taux d'inversion dépend aussi de l'énergie et de la densité des électrons créés durant la décharge, ainsi que des sections efficaces d'excitation, dans le cas tout au moins où il s'agit d'une excitation directe de la molécule à partir de son état fondamental. Ces sections efficaces sont comprises entre $10^{-16}$ et $10^{-17} \mathrm{~cm}^{2}$ et les calculs réalisés pour $\mathrm{N}_{2}$ et $\mathrm{H}_{2}[20,28]$ indiquent que la densité de courant dans le gaz doit être supérieure à $10^{4} \mathrm{~A} \mathrm{~cm}^{-2}$ pour que l'inversion soit voisine de $1 \%$ de la population totale. D'autre part le spectre d'énergie des électrons s'étendant de $0,1 \mathrm{eV}$ à $100 \mathrm{eV}$ environ, seule une partie de ceux-ci participera directement à l'excitation de la molécule jusqu'à l'état supérieur laser.

Pour réaliser de telles conditions une solution est de créer une impulsion de courant haute-tension entre deux électrodes plongeant dans le gaz. Les caractéristiques précises de cette impulsion doivent être ajustées dans chaque cas. Une grandeur repère est le rapport $E / p$ du champ électrique sur la pression totale du gaz. Ainsi pour $\mathrm{N}_{2}, E / p$ doit être $\simeq 100-200 \mathrm{~V} \mathrm{~cm}^{-1}$ torr $^{-1}$, pour $\mathrm{H}_{2}, \mathrm{D}_{2}, \mathrm{HD}, E / p \simeq 500 \mathrm{~V}^{-1} \mathrm{~cm}^{-1}$ torr $^{-1}$, tandis que pour $\mathrm{XeF}$ ou $\mathrm{KrF}$, il suffit que

$E / p \simeq 10-20 \mathrm{~V} \mathrm{~cm}^{-1}$ torr $^{-1}$.

Le montage de référence proposé par Shipman [15] est apparenté au montage Blumlein, classique en électronique des impulsions [61] et il est adapté au pompage de grandes longueurs amplificatrices. Dans ce montage (Fig. 7) chacune des deux électrodes plongeant dans le gaz constitue en même temps l'une des

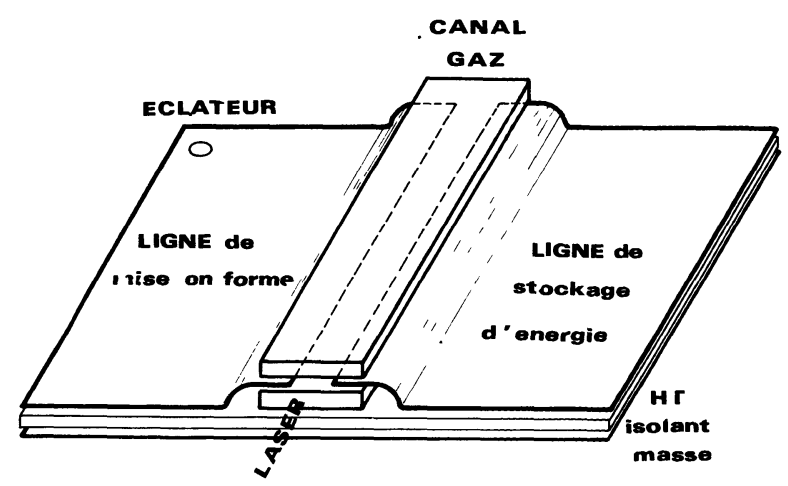

Fig. 7. - Schéma de principe d'un laser UV à pompage électronique transverse par décharge. 
armatures de deux lignes électriques de transmission. Ces lignes ont deux fonctions : d'une part elles constituent des éléments de stockage d'énergie de capacité $C_{0}$, d'autre part leur forme permet de transporter cette énergie, sous faible impédance $Z_{0}$ à travers le gaz, jusqu'à un interrupteur constitué par un éclateur ou un thyratron. La théorie du montage Blumlein nous indique que la tension appliquée au gaz est double de la tension de charge du système lorsque la résistance du gaz est égale à $2 Z_{0}$. Ce schéma idéalisé n'est pas transposable directement à la structure du laser et on doit considérer celle-ci comme un compromis entre le montage Blumlein et l'association de condensateurs de part et d'autre du canal. Plusieurs analyses ont été proposées, la plus élaborée étant certainement celle de Fitzsimmons et coll. [62].

L'intérêt des molécules excimères a conduit plus récemment à un nouveau développement de cette technologie. En particulier il a été démontré avec succès que l'addition au système de décharge principale d'un système de préionisation du gaz permettait d'accroître considérablement le rendement total d'émission [63, 64]. Deux types de préionisation ont été testés, l'un électronique, l'autre photonique. Dans les deux cas l'impulsion de préionisation doit précéder l'impulsion de la décharge principale, d'un temps voisin de $1 \mu \mathrm{s}$.

Les performances énergétiques de ces lasers sont indiquées tableau VII. On notera d'une part le nombre relativement important de longueurs d'onde du spectre couvertes actuellement ainsi que la diversité des résultats liée principalement à des détails technologiques.
D'une façon plus générale on remarquera que le domaine d'énergie émise couvert par ces lasers est compris entre 1 et $100 \mathrm{~mJ}$ et les durées d'émission sont au plus de 10 ns. Couvrant à la fois les domaines du proche UV et de l'UV du vide, leur domaine d'application est relativement étendu : pompage de colorants, séparation isotopique, diagnostique de plasmas, pompage de nouveaux atomes et molécules lasers, effet Raman stimulé, étude des caractéristiques fondamentales de la matière, etc...

4. Conclusion. - Les récents développements technologiques de ce type de laser ouvrent des perspectives très intéressantes. La preuve en est que des appareils répondant aux définitions que nous avons données, sont déjà commercialisés. Ils présentent un premier avantage : celui d'offrir un certain nombre de raies d'émission par simple changement du gaz ou du mélange de gaz. Un second avantage est sa relative compacité et sa mobilité qui le rend propre à des applications aussi bien en laboratoire que dans l'industrie.

On a assisté simultanément à un regain d'intérêt pour les études fondamentales des molécules, en particulier des molécules excimères, dont la connaissance jusqu'à présent était extrêmement réduite. A également été mise en valeur l'étude des processus fondamentaux des réactions cinétiques compte tenu de leur importance pour la connaissance et la maîtrise de l'émission stimulée dans un grand nombre de cas.

Nul doute également que de nouvelles émissions lasers dans l'UV seront mises en évidence dans un proche avenir, complétant ainsi le spectre des raies

Tableau VII. - Caractéristiques des principales émissions UV. lasers obtenues à partir du pompage par décharge de molécules diatomiques.

\begin{tabular}{|c|c|c|c|c|c|}
\hline Molécules & $\begin{array}{c}\text { Longueurs } \\
\text { d'onde lasers } \\
(\AA)\end{array}$ & Mélanges & $\begin{array}{l}\text { Energie laser } \\
\text { par impulsion } \\
(\mathrm{mJ})\end{array}$ & $\begin{array}{c}\text { Durée } \\
\text { d'émission } \\
\left(10^{-9} \mathrm{~s}\right)\end{array}$ & Références \\
\hline - & - & - & - & . - & - \\
\hline $\mathrm{XeF}$ & $\left.\begin{array}{ll}3 & 490 \\
3 & 510 \\
3 & 530\end{array}\right\}$ & $\begin{array}{l}\mathrm{Ar}: \mathrm{Xe}: \mathrm{F}_{2} \text { ou } \mathrm{NF}_{3} \\
\mathrm{He}: \mathrm{Xe}: \mathrm{F}_{2} \text { ou } \mathrm{NF}_{3} \\
\mathrm{Ne}: \mathrm{Xe}: \mathrm{NF}_{3}\end{array}$ & $\leqslant 100$ & $10-20$ & $\begin{array}{l}{[39,40,63,64]} \\
{[66-70]}\end{array}$ \\
\hline $\mathrm{N}_{2}$ & $\begin{array}{l}3576 \\
3371 \\
3159\end{array}$ & $\begin{array}{l}\mathrm{N}_{2} \\
\mathrm{~N}_{2}: \mathrm{SF}_{6}\end{array}$ & $\leqslant 10$ & $3-10$ & {$[17,62]$} \\
\hline $\mathrm{XeCl}$ & 3080 & $\mathrm{He}: \mathrm{Xe}: \mathrm{Cl}_{2}$ ou $\mathrm{BCl}_{3}$ & $\sim 1$ & 10 & [71] \\
\hline $\mathrm{Br}_{2}$ & 2920 & $\mathrm{Br}_{2}$ & & & {$[58]$} \\
\hline $\mathrm{KrF}$ & 2490 & $\begin{array}{l}\text { Ar ou He : Kr : } F_{2} \\
\text { ou } \mathrm{NF}_{3} \text { ou } N_{2} F_{4}\end{array}$ & $\leqslant 250$ & 10 & $\begin{array}{l}{[66,67,69]} \\
{[72-75]}\end{array}$ \\
\hline $\mathrm{KrCl}$ & 2229 & $\mathrm{Kr}: \mathrm{Cl}_{2}$ & $\leqslant 2$ & $\sim 10$ & {$[71,76]$} \\
\hline $\mathrm{CO}$ & 1810 à 1970 & $\mathrm{CO}$ & $\sim 10^{-4}$ & - & [13] \\
\hline $\operatorname{ArF}$ & 1930 & $\mathrm{He}: \mathrm{Ar}: \mathrm{F}_{2}$ ou $\mathrm{NF}_{3}$ & $\leqslant 100$ & $\sim 10$ & {$[72,76,77]$} \\
\hline $\mathrm{ArCl}$ & 1750 & $\mathrm{He}: \mathrm{Ar}: \mathrm{Cl}_{2}$ & 0,2 & 10 & [76] \\
\hline $\left.\begin{array}{l}\mathrm{H}_{2}, \mathrm{D}_{2} \\
\mathrm{HD}\end{array}\right\}$ & $\sim 1600$ & $\mathrm{H}_{2}, \mathrm{D}_{2}, \mathrm{HD}$ & 1 & $\leqslant 1$ & {$[28-35]$} \\
\hline $\mathrm{H}_{2}$ & $\sim 1200$ & $\mathrm{H}_{2}$ & $10^{-3}$ & $\leqslant 1$ & [36] \\
\hline
\end{tabular}


déjà connues et offrant en même temps de nouvelles perspectives d'applications.

Enfin, la voie d'une exploitation réfléchie des possibilités d'émission laser UV à partir de molécules triatomiques est également ouverte. Ainsi par exemple, la molécule $\operatorname{Ar}_{2} \mathrm{~F}$ [65] est susceptible de fournir des émissions intenses à $2680 \AA$ et $2740 \AA$ à partir de transitions entre l'état lié $2^{2} \mathrm{~B}_{2}$ et les états répulsifs
$1{ }^{2} \mathrm{~A}_{1}$ et $1{ }^{2} \mathrm{~B}_{2}$. De plus, les durées de vie naturelles de ces transitions ont été estimées à 128 ns. Dans le même esprit, on peut penser que la série des molécules excimères $\mathrm{Ar}_{2} \mathrm{Cl}, \mathrm{Ar}_{2} \mathrm{Br}$ et $\mathrm{Ar}_{2} \mathrm{I}$ est susceptible de fournir des émissions stimulées.

Ce nouveau champ d'études est donc très largement ouvert et peut rapidement s'étendre avec la collaboration de la physique moléculaire et de la physico-chimie.

\section{Bibliographie}

[1] Maiman, T. H., Nature 187 (1960) 493.

[2] Kennedy, R. S., Proc. IEEE 58 (1970) 1651-65.

[3] LERNER, R. M., M.I.T. Lincoln Laboratory quarterly technical summary (M.I.T. Lincoln Lab., Lexington) 15 juin 1972, p. 11-16.

[4] KidDer, R. E., Physics of High Energy Density, Caldirda and Knoepfel (Ed. New York, Academic Press) 1971.

[5] Voir par exemple : Weisskopf, V., Z. Phys. 34 (1933) 1 ; FAno, U., Phys. Rev. 131 (1963) 259 ;

Berman, P. R., Phys. Rev. A 6 (1972) 2157.

[6] Griem, H. R., Plasma Spectroscopy (New York : McGrawHill) 1964.

[7] Mark, H., The Physics of Electronic and Atomic Collisions (VII ICPEAC), T. R. Govers et F. J. de Heer, Ed. Amsterdam : North-Holland (1972).

[8] Handbook of Lasers, R. J. Pressley Ed., The Chemical Rubber Co, Cleveland (USA) 1971.

[9] Wilson, J., Appl. Phys. Lett. 8 (1966) 159 ;

TARG, R., IEEE J. Quant. Elect. (1972) 726.

[10] Hoffman, J. M., Hays, A. K., Tisone, G. C., Appl. Phys. Lett. 28 (1976) 538.

[11] Rothe, D. E. et TaN, K. O., Appl. Phys. Lett. 30 (1977) 152.

[12] Herzberg, G., Molecular Spectra and Molecular structure : Spectra of diatomic molecules (Princeton, N.J., Van Nostrand Ed.) 1950.

[13] Hodgson, R. T., J. Chem. Phys. 55 (1971) 5378.

[14] HEARD, R. G., Nature 200 (1963) 667.

[15] Shipman, J. D., Appl. Phys. Lett. 10 (1967) 3.

[16] Garavaglia, M., Gallardo, M., Massone, D. A., Phys. Lett. 28A (1969) 787.

[17] GirardeaU-Montaut, J. P., Nouv. Rev. Optique 5 (1974) 367.

[18] GreEN, A. E. S., The middle Ultra-violet : its sciences and technology (J. Wiley Ed., New York) 1966, 170.

[19] Peterson, L. R., Prasad, S. S., Green, A. E. S., Can. J. Chem. 47 (1969) 1774

[20] Girardeau-Montaut, J. P., Girardeau-Montaut, C., Nouv. Rev. Optique 5 (1974) 179.

[21] Cartwright, D. C., Phys. Rev. A 2 (1970) 1331

[22] Kaslin, V. M., Petrash, G. G., Sov. Phys. JetP 27 (1968) 561 .

[23] Girardeau-Montaut, J. P., Onde Elect. 54 (1974) 456.

[24] Girardeau-Montaut, J. P., Thèse Doctorat d'Etat (1972), Univ. de Clermont.

[25] Willet, C. S., Litynski, D. M., Appl. Phys. Lett. 26 (1975) 118 ;

Suchard, S. N., Sutton, D. G., Heidner, R. F., IEEE J. Quant. Electron. QE 11 (1975) 908 ;

Akins, R. P., Shao Chin Lin, Appl. Phys. Lett. 28 (1976) 221.

[26] Bazhulin, P. A., Knyazev, I. N., Petrash, G., Sov. Phys. JETP 23 (1965) 649

[27] Geiger, J., Z. Phys. 181 (1964) 413 ;

Lassettre, E. N., Jones, E. A., J. Chem. Phys. 40 (1964) 1222 ; Bethe, H., Ann. Phys. 5 (1930) 325.

[28] Knyazev, I. N., Letokhov, V. S., Movshev, V. G., IEEE J. Quant. Electron. QE 11 (1975) 805.

[29] Hodgson, R. T., Phys. Rev. Lett. 25 (1970) 494.
[30] Waynant, R. W., Shipman, J. D., Elton, R. C., Ali, A. W., Appl. Phys. Lett. 17 (1970) 383.

[31] Hodgson, R. T., Dreyfus, R. W., Phys. Lett. 38A (1972) 213.

[32] Waynant, R. W., Shipman, J. D., Elton, R. C., Ali, A. W., Proc. IEEE 59 (1971) 679.

[33] Waynant, R. W., Ali, A. W., Julienne, P. S., J. Appl. Phys. 42 (1971) 3406.

[34] Girardeau-Montaut, J. P., Chaumat, J. P., GirardeauMonTAUT, C., Experimental molecular Hydrogen V.U.V. laser. Ve intern. Conf. Montpellier (France) 5 au 9 Sept. 1977.

[35̄] Hodgson, R. T., Dreyfus, R. W., Phys. Rev. Lett. 28 (1972) 536.

[36] Waynant, R. W., Phys. Rev. Lett. 28 (1972) 553.

[37] Houtermans, F. G., Helv. Phys. Acta 33 (1960) 933.

[38] Basov, N. G., Danilychev, V. A., Popov, Yu. M., КноDEVICH, D. D., JETP Lett. 9 (1970) 329.

[39] Christensen, C. P., Appl. Phys. Lett. 30 (1977) 483 ; Johnson, P. M., Keller, N., Turner, R. E., Appl. Phys. Lett. 32 (1978) 291.

[40] Champagne, L. F., Harris, N. W., Appl. Phys. Lett. 31 (1977) 513.

[41] Flannery, M. R., Yang, T. P., Appl. Phys. Lett. 32 (1978) 327.

[42] Eden, J. G., Searles, S. K., Appl. Phys. Lett. 29 (1976) 356.

[43] Hawryluk, A. M., Mangano, J. A., Jacob, J. H., Appl. Phys. Lett. 31 (1977) 164.

[44] Dunning, T. H., Hay, P. J., Appl. Phys. Lett. 28 (1976) 649.

[45] Mangano, J. A., Jacob, J. H., Rokni, M., Hawryluk, A., Appl. Phys. Lett. 31 (1977) 26.

[46] McCusker, M. V., Hill, R. M., Huestis, D. L., Lorents, D. C., Gutcheck, R. A., Nakano, H. H., Appl. Phys. Lett. 27 (1975) 363.

[47] Mulliken, R. S., J. Chem. Phys. 55 (1971) 288.

[48] Tellinghuisen, J., Chem. Phys. Lett. 29 (1974) 359.

[49] Hays, A. K., Hoffman, J. M., Tisone, G. C., Chem. Phys. Lett. 39 (1976) 353.

[50] Hays, A. K., Hoffman, J. M., Tisone, G. C., « Molecular iodine laser near $3400 \mathrm{~A}$ " presented at 2nd Summer Colloquium on Electron. Transition Lasers, Woodshole, Mass., 17-19 Sept. 1975

[51] Ewing, J. J., Brau, C. A., Appl. Phys. Lett. 27 (1975) 557.

[52] Bradford, R. S., Jr, Ault, E. R., Bhaumik, M. L., Appl. Phys. Lett. 27 (1975) 546.

[53] Fisk, G. A., Mahan, B. H., Parks, E. K., J. Chem. Phys, 47 (1967) 2649.

[54] Wodarczyk, F. J., Schlossberg, H. R., J. Chem. Phys. 67 (1977) 4476.

[55] Tellinghuisen, J., Chem. Phys. Lett. 49 (1977) 485.

[56] Swingle, J. C., Turner, C. E. Jr, Murray, J. R., George, E. V., Krupke, W. F., Appl. Phys. Lett. 28 (1976) 387.

[57] Murray, J. R., Swingle, J. C., Turner, C. E. Jr, Appl. Phys. Lett. 28 (1976) 530.

[58] Ewing, J. J., Jacob, J. H., Mangano, J. A., Brown, H. A., Appl. Phys. Lett. 28 (1976) 656. 
[59] Chen, C. H., Payne, M. G., Appl. Phys. Lett. 28 (1976) 219. [60] Okabe, H., Laufer, A. H., Ball, J. J., J. Chem. Phys. 55 (1971) 373.

[61] Ramo, S., Whinnery, J. R., VAn Duzer, T., Fields and waves Communication electronics (John Wiley Ed., New York) 1965 .

[62] Fitzsimmons, W. A., Anderson, L. W., Riedhauser, C. E., VRTILEK, J. M., IEEE J. Quant. Electron. QE 12 (1976) 624.

[63] Burnham, R., Harris, N. W., Djeu, N., Appl. Phys. Lett. 28 (1976) 86.

[64] Wang, C. P., Mirels, H., Sutton, D. G., Suchard, S. N., Appl. Phys. Lett. 28 (1976) 326.

[65] Wadt, W. R., Hay, P. J., Appl. Phys. Lett. 30 (1977) 573.

[66] Burnham, R., Powell, F. X., Djeu, N., Appl. Phys. Lett. 29 (1976) 30.

[67] Burnham, R., Djeu, N., Appl. Phys. Lett. 29 (1976) 707.

[68] Sarjeant, W. J., Alcock, A. J., Leopold, K. E., Appl. Phys. Lett. 30 (1977) 635.

[69] Wang, C. P., Appl. Phys. Lett. 29 (1976) 103.

[70] Hasson, V., Lee, C. M., Exberger, R., Billman, K. W., Rowley, P. D., Appl. Phys. Lett. 31 (1977) 167.
[71] IshChENKo, V. N., Lisitsyn, V. N., RAZHEV, A. M., Optics commun. 21 (1977) 30.

[72] Loree, T. R., Butterfield, K. B., Barker, D. L., Appl. Phys. Lett. 32 (1978) 171.

[73] Sutton, D. G., Suchard, S. N., GibB, O. L., Wang, C. P., Appl. Phys. Lett. 28 (1976) 522.

[74] Andrews, A. J., Kearsley, A. J., WebB, C. E., Haydon, S. C., Optics commun. 20 (1977) 265.

[75] Sarjeant, W. J., Alcock, A. J., Leopold, K. E., IEEe Quant. Electron. QE 14 (1978) 177.

[76] Waynant, R. W., Appl. Phys. Lett. 30 (1977) 287.

[77] Kudrautsev, Y. A., Kuzmina, N. P., Appl. Phys. 13 (1977) 107.

[78] Lacina, W. B., Cohn, D. B., Appl. Phys. Lett. 32 (1978) 106.

[79] Shui, V. H., Appl. Phys. Lett. 31 (1977) 50.

[80] JaCob, J. H., Rokni, M., Mangano, J. A., Brochu, R., Appl. Phys. Lett. 32 (1978) 109.

[81] Brashears, H. C. Jr, Setser, D. W., Des Marteau, D., Chem. Phys. Lett. 48 (1977) 84.

[82] Rokni, M., Jacob, J. H., Mangano, J. A., Brochu, R., Appl. Phys. Lett. 32 (1978) 223. 